\title{
BMJ Open Development and validation of clinical prediction models for mortality, functional outcome and cognitive impairment after stroke: a study protocol
}

Marion Fahey, ${ }^{1}$ Anthony Rudd, ${ }^{1,2}$ Yannick Béjot, ${ }^{3}$ Charles Wolfe, ${ }^{1,4}$ Abdel Douiri ${ }^{1,4}$

To cite: Fahey M, Rudd A, Béjot $\mathrm{Y}$, et al. Development and validation of clinical prediction models for mortality, functional outcome and cognitive impairment after stroke: a study protocol. BMJ Open 2017;7:e014607. doi:10.1136/ bmjopen-2016-014607

- Prepublication history for this paper is available online. To view these files please visit the journal online (http://dx.doi org/10.1136/bmjopen-2016014607).

Received 13 0ctober 2016 Revised 8 February 2017 Accepted 23 March 2017

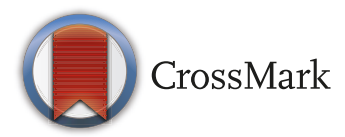

${ }^{1}$ Division of Health and Social Care Research, King's College London, London, UK

${ }^{2}$ National Institute for Health Research (NIHR) Biomedical Research Centre, Guy's and St Thomas' NHS Foundation Trust, London, UK

${ }^{3}$ Registre Dijonnais des AVC, EA4184, Dijon, France

${ }^{4}$ National Institute for Health Research (NIHR) Collaboration for Leadership in Applied Health Research and Care (CLAHRC),

South London at King's College Hospital NHS Foundation Trust, London, UK

Correspondence to

Marion Fahey;

marion.fahey@kcl.ac.uk

\section{ABSTRACT}

Introduction Stroke is a leading cause of adult disability and death worldwide. The neurological impairments associated with stroke prevent patients from performing basic daily activities and have enormous impact on families and caregivers. Practical and accurate tools to assist in predicting outcome after stroke at patient level can provide significant aid for patient management. Furthermore, prediction models of this kind can be useful for clinical research, health economics, policymaking and clinical decision support.

Methods 2869 patients with first-ever stroke from South London Stroke Register (SLSR) (1995-2004) will be included in the development cohort. We will use information captured after baseline to construct multilevel models and a Cox proportional hazard model to predict cognitive impairment, functional outcome and mortality up to 5 years after stroke. Repeated random subsampling validation (Monte Carlo cross-validation) will be evaluated in model development. Data from participants recruited to the stroke register (2005-2014) will be used for temporal validation of the models. Data from participants recruited to the Dijon Stroke Register (1985-2015) will be used for external validation. Discrimination, calibration and clinical utility of the models will be presented.

Ethics Patients, or for patients who cannot consent their relatives, gave written informed consent to participate in stroke-related studies within the SLSR. The SLSR design was approved by the ethics committees of Guy's and St Thomas' NHS Foundation Trust, Kings College Hospital, Queens Square and Westminster Hospitals (London). The Dijon Stroke Registry was approved by the Comité National des Registres and the InVS and has authorisation of the Commission Nationale de l'Informatique et des Libertés.

\section{INTRODUCTION}

Stroke is one of the most common causes of serious adult physical disability and the third most common cause of death worldwide. ${ }^{1}$ Despite the introduction of effective treatments for acute stroke, early rehabilitation and secondary prevention, the majority of stroke survivors have medical comorbidities, physical
Strengths and limitations of this study

- First prognostic tool in stroke to follow, a priori, the Prognosis Research Strategy (PROGRESS) framework, and Transparent Reporting of a multivariable prediction model for Individual Prognosis Or Diagnosis (TRIPOD) reporting guidelines for prognostic research.

- Specifies statistical analysis plan and informative levels of tool performance to increase transparency of results and the final report.

- The proposed study predicts outcomes longitudinally, which may be more reflective of clinical needs than predictions made at predefined time points.

- The proposed study is restricted by the use of predictor variables measured in previous data sets and the limitations of these measures. The external validation is not independent.

and/or cognitive impairments that require ongoing active assessment and management. ${ }^{2}$ Stroke can be seen as a chronic condition, spanning not only the incident event and formal rehabilitation but the rest of the patient's life. Rehabilitation from stroke requires a sustained, coordinated effort from informed multidisciplinary teams (MDTs), as well as patients and carers, both in the clinical setting and in the community. ${ }^{3}$ MDTs and patients make numerous decisions on the basis of an estimated probability that a specific event will occur in the future. These predictions are used for planning lifestyle or therapeutic decisions on the basis of the risk of developing a particular outcome or state of health. ${ }^{4-6}$ More recently such estimates are used to risk-stratify participants in therapeutic intervention trials and case-mix classifications. ${ }^{78}$

Information from a single predictor is often insufficient to provide reliable estimates of prognostic probabilities or risks, particularly 
in complex patients, for example those with comorbidities. $^{9} 10$ Therefore probability estimates are commonly based on combining information from multiple predictors to form a multivariable clinical prediction model $(\mathrm{CPM}) .^{11} 12$

CPMs are abundant in prognostic research literature, but few are implemented or used in routine clinical practice. ${ }^{13}$ One explanation for this is that, although many models have been developed, ${ }^{145}$ they have limited utility for clinical applications, particularly in a long-term care setting. Progression or regression of disease is highly variable both over time and between individuals. ${ }^{16}$ Current CPMs typically estimate risk at predefined time points. With variability in mind, time series data and methods may be more appropriate for accurately capturing recovery, particularly when the aim is planning immediate and long-term care simultaneously for individual patients.

There is generally a lack of confidence among clinicians in applying risk scores in practice. Many believe there is the lack of sufficient evidence to demonstrate the reproducibility and transportability of the model in a different population. ${ }^{17}$ To be considered useful, a risk score should be clinically credible, accurate (well calibrated with good discriminative ability) and have generality (be externally validated). ${ }^{17}$

We have evaluated the accuracy of existing models in predicting stroke outcomes by systematic review of literature and meta-analysis. The systematic review and meta-analysis concluded that existing models have much potential and advised to build on previous work, as opposed to designing new models, in developing CPMs suitable for the long-term care setting. ${ }^{15}$ Models predicting outcomes longitudinally, as opposed to predefined time points, may best support patient management and so we will build on work by colleagues in this area. ${ }^{18} 19$

\section{OBJECTIVES}

\section{Primary objectives}

The primary objectives were to develop and internally validate prediction models in patients with ischaemic stroke for functional outcome, survival and cognitive impairment at 5 years, and to externally validate and update these models through an external data set of patients with ischaemic stroke.

\section{Secondary objectives}

The secondary objectives were to assess deviations of predicted recovery curves, to investigate why patients with stroke regain different recovery that plateaued at different levels, and to derive and validate scoring system for classifying risks (case-mix classification) from these patient-centred predictive models.

\section{METHODS}

This cohort study is informed by the Prognosis Research Strategy (PROGRESS) framework and recommendations by authors in the field. We will report the study protocol using the Transparent Reporting of a multivariable prediction model for Individual Prognosis Or Diagnosis (TRIPOD) statement for prediction studies. ${ }^{20}$

\section{Source of data}

The study is a prospective longitudinal cohort study. The data source is the South London Stroke Register (SLSR), an ongoing, prospective, population-based stroke register set up in January 1995 recording all first-ever strokes in patients of all ages for an inner area of South London. The methods of the SLSR have been described previously in detail ${ }^{2}$ and are summarised here. Stroke was defined according to the WHO criteria and Oxfordshire Community Stroke Project Classification. Multiple overlapping sources of information are employed. Data from 1995 to 2016 will be used for model derivation and internal validation.

\section{Participants}

Patients admitted to hospitals serving the study area (two teaching hospitals within and three hospitals outside the study area) are identified by regular reviews of acute wards admitting patients with stroke, and national data on patients admitted to any hospital in England and Wales with a diagnosis of stroke are screened for additional patients. All general practitioners ( $\mathrm{n}=699$ (2011)) within and on the borders of the study area are contacted regularly and asked to notify the SLSR of patients with stroke. Referral of non-hospitalised patients with stroke to a neurovascular outpatient clinic (from 2003) or domiciliary visit to patients by the study team is also available to general practitioners. Community therapists are contacted every 3 months. Death certificates are checked regularly. Patients are assessed at the stroke onset, 3 months and annually after stroke. Participants receive treatments in line with the UK national clinical guidelines for stroke. Completeness of case ascertainment has been estimated at $88 \%$ by a multinomial logit capture-recapture model using the methods described in detail elsewhere. ${ }^{21}$

\section{Outcome}

The outcomes of interest are mortality measured as time to event, functional outcome measured using the Barthel Index (BI) and cognitive impairment measured using the Mini-Mental State Exam (MMSE) or Abbreviated Mental Test (AMT), measured at baseline, 3 months and annually up to 5 years after stroke. Specially trained nurses and field workers collect all data prospectively whenever feasible. A study doctor verifies the diagnosis of stroke. Patients are examined within 48 hours of referral to the SLSR where possible. Follow-up data are collected by validated postal or face-to-face instruments with patients and/or their carers. Outcome definition and measurement method are the same for all patients. Data collection is carried out by a third party, uninvolved in this study.

\section{Predictors}

Candidate predictors to be considered in the prediction models will be based on predictor variables 
as identified in previous systematic reviews in this field, ${ }^{1522}$ ease and reliability of measurement in clinical setting and theoretical association with the progression of outcomes. The number of variables required to ensure adequate power based on our target sample size (see below), to avoid overfitting and to encourage parsimony and applicability of the model in clinical practice will also be considered. Number, type, definition, method for measurement and handling of candidate predictors in the modelling are listed in the data supplement. All predictors are measured on patient presentation. Data collection is carried out by a third party, not involved in this research.

\section{Sample size}

Cumulative survival up to 10 years after stroke is estimated at $63.7 \%, 42.8 \%$ and $24.0 \%$, surviving up to 1 , 5 and 10 years, respectively. ${ }^{2}$ Disability is estimated on average at $11 \%$ (10-year average and standardised to European population in the SLSR). ${ }^{2}$ Current SLSR data (1995-2015) estimate disability (BI $>15)$ at $24.6 \%$, $23.7 \%$ and $26.3 \%$ at 1,5 and 10 years, respectively. Cognitive impairment (MMSE $<24$ ) is estimated at approximately $22 \%$ in the first 5 years following stroke (age-standardised to European population) ${ }^{23}$ Current SLSR data (1995-2015) estimate cognitive impairment at $29.8 \%, 28.9 \%, 28.6 \%$ at 1,5 and 10 years, respectively. Rules of thumb for fitting multivariate models suggest that 10 events for every variable (EPV) are required to avoid overfitting in model development studies. Although we are within these limitations, the use of this rule can result in small sample sizes, which may lead to overfitting and optimism. It is recommended that EPV should be data-driven. ${ }^{24}$ Sample size calculations described by Jinks ${ }^{25}$ will be used for survival analysis. Simulation will be used to determine appropriate sample size for prediction models for functional outcome and cognitive impairment.

\section{Missing data}

Missing data are inevitable in studies with long follow-up and may lead to bias and imprecision. Multiple imputation will be used to impute missing values, under a missing at random assumption, so as to reduce bias and avoid excluding participants from the analysis. ${ }^{26-30}$ Imputations typically break down when missing data are excessive; therefore, data with more than $80 \%$ missing data will be excluded.

\section{Statistical analysis methods}

We will develop three CPMs for the outcomes: (1) mortality, (2) functional outcome and (3) cognitive impairment up to 5 years after stroke. The start point is time of stroke and end point is 5 years post stroke.

For predictor selection during multivariate modelling, a variable selection and shrinkage procedure will be used to decide which of the identified candidate predictor variables should be included in the final prediction model. Continuous variables will be kept as continuous (rather than say dichotomising) to avoid loss of power. Non-linear trends will be considered using fractional polynomials and the multivariable fractional polynomial procedure. Clinically meaningful interactions (eg, time) will be included in the model.

We will assess internal validity with a bootstrapping procedure for a realistic estimate of the performance of prediction models in similar future patients. ${ }^{512}$ The bootstrap validation approach uses all of the data to develop the prediction model and provides a mechanism to account for model overfitting or uncertainty in the entire model development process, thereby quantifying any optimism in the final prediction model. Also, it provides for estimating a shrinkage factor that can be used to adjust the regression coefficients and apparent performance for optimism, such that better performance will be obtained in subsequent model validation studies and applications. ${ }^{31}$

External validity will be assessed using data form the Dijon Stroke Register (DSR). Patients will be classified using the estimates of the previously developed models and performance assessed. Subgroup analyses will be carried out to assess deviations from predicted recovery trajectories and investigate why patients with stroke regain different recovery that plateaued at different levels.

\section{Risk groups}

Although risk groups (eg, 'high risk', 'moderate risk', 'low risk') may make models more accessible, no risk groups will be created. There is no clear consensus on how to create risk groups or how many groups to use. ${ }^{31}$ There are concerns that use of risk groups may not be in the best interest of patients and may become standard, although lacking any rational. ${ }^{31}$ Also, the simplification of predicted probabilities assumes risk is the same for all individuals within that category.

\section{Development versus validation}

Data from the DSR will be used for external validation; its methods have been described previously in detail ${ }^{32-34}$ and both data sets are contrasted here. The DSR and SLSR are population-based registers; multiple overlapping sources of notification are used and stroke is defined according to WHO criteria for both. Specially trained field workers collect all data. This includes sociodemographic factors, disease characteristics, patient history and cardiovascular risk factors in both registers. DSR participants are followed up at time of stroke and yearly thereafter; these questionnaires are administered in outpatient clinics or conducted telephonically by clinical nurses. Follow-up procedures in SLSR are similar, but participants are followed up at 3 months also. In both registers, survival is measured using national data, cognitive status is measured using either the AMT or the MMSE, and disability is measured using BI. 


\section{REPORTING OF RESULTS}

\section{Participants}

The flow of participants through the study, including the number of participants with and without the outcome, and a summary of the follow-up time will be described. The characteristics of the participants, including the number of participants with missing data for predictors and outcome for both development and validation cohorts, will be provided.

\section{Model development}

The number of participants and outcome events in each analysis will be presented, as well as the unadjusted association between each candidate predictor and outcome.

\section{Model specification}

The full prediction model for each outcome will be presented, including all regression coefficients, and model intercept and baseline survival. Once a final model is identified, methods will be applied to simplify and adapt the presentation of the model to a scoring system to facilitate its application in practice at a later date. An explanation of how to use the model and scoring system will also be presented.

\section{Model performance}

Model performance assessment has been designed using the framework described by Steyerberg and colleagues. ${ }^{35}$ Model performance will be assessed in derivation and validation data sets. For model development studies, we are primarily interested in discrimination, because the model will be well calibrated (on average) by definition when smoothing methods are used. In validation studies, assessment of both discrimination and calibration is fundamental. ${ }^{35}{ }^{36}$ Calibration of the derived models will be measured using calibration plot and Hosmer-Lemeshow test or counterpart test for the survival model. ${ }^{37}{ }^{38}$ Furthermore, they convey no indication of magnitude or direction of any miscalibration; hence, calibration plots will also be presented. Calibration plots will also be evaluated in relation to key predictors/subgroups. Discrimination of the derived models will be measured using the concordance statistic and CIs (c-statistic). The c-index is identical to the area under the receiver operating characteristic curve for models with binary end points and can be generalised for time-to-event (survival) models accounting for censoring. A benchmark level of discrimination was determined in our meta-analysis for mortality and functional outcome. On the basis of the reference standard values in statistics literature ${ }^{39}$ and those of previous work in stroke, we will consider area under the curve values of greater than 0.8 to be acceptable for these outcomes. Classification measures (eg, sensitivity, specificity, predictive values, net reclassification improvement) will be presented and cut points selected a priori. Decision curve analysis will be undertaken to assess clinical utility. ${ }^{40}$

\section{Model updating}

We will update the model if it shows poor performance in external data (DSR) by recalibration or revision methods depending on discrimination performance (c-statistics $<0.80$ ). If model is updated, updating approaches recommended by Steyerberg $e t \mathrm{ll}^{13}$ (model recalibration, model revision, model extension) will be adopted as appropriate.

\section{CONCLUSION}

We have described the methods and statistical analysis plan to develop and to validate a family of CPMs for stroke over the long term. To our knowledge, this tool will be the first of its kind in stroke to follow, a priori, the PROGRESS framework and TRIPOD reporting guidelines for prognostic research. Importantly for secondary prevention, the tool will be developed specifically to predict the progression of disease and to identify those at high risk of an adverse outcome. Results coming from this study will be interpreted for both clinical and research purposes.

Contributors MF: drafting of article. AR: critical revising of the article. YB: critical revising of the article. CW: critical revising of the article. $A D$ : critical revising of the article.

Competing interests None declared.

Ethics approval The SLSR design was approved by the ethics committees of Guys and St Thomas NHS Foundation Trust, Kings College Hospital, Queens Square and Westminster Hospitals (London). The Dijon Stroke Registry was approved by the Comit National des Registres and the InVS and has authorisation of the Commission Nationale de Informatique et des Liberts.

Provenance and peer review Not commissioned; externally peer reviewed.

Open Access This is an Open Access article distributed in accordance with the terms of the Creative Commons Attribution (CC BY 4.0) license, which permits others to distribute, remix, adapt and build upon this work, for commercial use, provided the original work is properly cited. See: http://creativecommons.org/ licenses/by/4.0/

(c) Article author(s) (or their employer(s) unless otherwise stated in the text of the article) 2017. All rights reserved. No commercial use is permitted unless otherwise expressly granted.

\section{REFERENCES}

1. Feigin VL, Forouzanfar MH, Krishnamurthi R, et al. Global and regional burden of stroke during 1990-2010: findings from the global burden of disease study 2010. Lancet 2014;383:245-55.

2. Wolfe CD, Crichton SL, Heuschmann PU, et al. Estimates of outcomes up to ten years after stroke: analysis from the prospective South London Stroke Register. PLoS Med 2011;8:e1001033.

3. Winstein CJ, Stein J, Arena R, et al. Guidelines for adult Stroke Rehabilitation and Recovery: a Guideline for Healthcare Professionals from the American Heart Association/American Stroke Association. Stroke 2016;47:e98-e169.

4. Moons KG, Altman DG, Vergouwe Y, et al. Prognosis and prognostic research: application and impact of prognostic models in clinical practice. BMJ 2009;338:b606.

5. Steyerberg EW. Clinical prediction models: a practical approach to development, validation, and updating. New York: Springer, 2009.

6. Wasson JH, Sox HC, Neff RK, et al. Clinical prediction rules. applications and methodological standards. N Engl J Med 1985;313:793-9.

7. Dorresteijn JA, Visseren FL, Ridker PM, et al. Estimating treatment effects for individual patients based on the results of randomised clinical trials. BMJ 2011;343:d5888.

8. Kattan MW, Vickers AJ. Incorporating predictions of individual patient risk in clinical trials. Urol Oncol 2004;22:348-52.

9. Riley RD, Hayden JA, Steyerberg EW, et al. Prognosis Research Strategy (PROGRESS) 2: prognostic factor research. PLoS Med 2013;10:e1001380.

10. Collins GS, Omar O, Shanyinde M, et al. A systematic review finds prediction models for chronic kidney disease were poorly reported 
and often developed using inappropriate methods. $J$ Clin Epidemiol 2013;66:268-77.

11. Royston P, Altman DG. External validation of a cox prognostic model: principles and methods. BMC Med Res Methodol 2013;13:33.

12. Harrell FE, Lee KL, Mark DB. Multivariable prognostic models: issues in developing models, evaluating assumptions and adequacy, and measuring and reducing errors. Stat $\mathrm{Med}$ 1996;15:361-87.

13. Steyerberg EW, Moons KG, van der Windt DA, et al. Prognosis Research Strategy (PROGRESS) 3: prognostic model research. PLOS Med 2013;10:e1001381.

14. Counsell C, Dennis M. Systematic review of prognostic models in patients with acute stroke. Cerebrovasc Dis 2001;12:159-70.

15. Fahey M, Crayton E, Charles W, et al. Oral abstracts: clinical prediction models for ischemic stroke recovery: a systematic review and Meta-Analysis. European Stroke Journal 2016;1:613-780.

16. Langhorne P, Bernhardt J, Kwakkel G. Stroke rehabilitation. Lancet 2011;377:1693-702.

17. Altman DG. Prognostic models: a methodological framework and review of models for breast cancer. Cancer Invest 2009;27:235-43.

18. Tilling K, Sterne JA, Wolfe CD. Multilevel growth curve models with covariate effects: application to recovery after stroke. Stat Med 2001;20:685-704.

19. Tilling K, Sterne JA, Rudd AG, et al. A new method for predicting recovery after stroke. Stroke 2001;32:2867-73.

20. Moons KG, Altman DG, Reitsma JB, et al. New Guideline for the Reporting of Studies developing, validating, or updating a multivariable clinical prediction Model: the TRIPOD statement. Adv Anat Pathol 2015:22:303-5.

21. Tilling K, Sterne JA, Wolfe CD. Estimation of the incidence of stroke using a capture-recapture model including covariates. Int $J$ Epidemiol 2001;30:1351-9.

22. Pendlebury ST, Rothwell PM. Prevalence, incidence, and factors associated with pre-stroke and post-stroke dementia: a systematic review and meta-analysis. Lancet Neurol 2009;8:1006-18.

23. Douiri A, Rudd AG, Wolfe CD. Prevalence of poststroke cognitive impairment: south London Stroke Register 1995-2010. Stroke 2013;44:138-45.

24. Ogundimu EO, Altman DG, Collins GS. Adequate sample size for developing prediction models is not simply related to events per variable. J Clin Epidemiol 2016;76:175-82.

25. Jinks R. Doctoral Thesis. Sample size for multivariable prognostic models. University College London; 2012.
26. Donders AR, van der Heijden GJ, Stijnen T, et al. Review: a gentle introduction to imputation of missing values. $J$ Clin Epidemiol 2006;59:1087-91.

27. Groenwold $\mathrm{RH}$, White IR, Donders AR, et al. Missing covariate data in clinical research: when and when not to use the missing-indicator method for analysis. CMAJ 2012;184:1265-9.

28. Janssen KJ, Vergouwe $\mathrm{Y}$, Donders AR, et al. Dealing with missing predictor values when applying clinical prediction models. Clin Chem 2009;55:994-1001.

29. Moons KG, Donders RA, Stijnen T, et al. Using the outcome for imputation of missing predictor values was preferred. J Clin Epidemiol 2006;59:1092-101.

30. Sterne JA, White IR, Carlin JB, et al. Multiple imputation for missing data in epidemiological and clinical research: potential and pitfalls. BMJ 2009;338:b2393.

31. Moons KG, Altman DG, Reitsma JB, et al. Transparent Reporting of a multivariable prediction model for individual prognosis or diagnosis (TRIPOD): explanation and elaboration. Ann Intern Med 2015;162:W1-73

32. Giroud M, Lemesle M, Quantin C, et al. A hospital-based and a population-based stroke registry yield different results: the experience in Dijon, France. Neuroepidemiology 1997;16:15-21.

33. Béjot $Y$, Daubail $B$, Jacquin $A$, et al. Trends in the incidence of ischaemic stroke in young adults between 1985 and 2011: the Dijon Stroke Registry. J Neurol Neurosurg Psychiatry 2014;85:509-13.

34. Lainay C, Benzenine E, Durier J, et al. Hospitalization within the first year after stroke: the Dijon stroke registry. Stroke 2015;46:190-6.

35. Steyerberg EW, Vickers AJ, Cook NR, et al. Assessing the performance of prediction models: a framework for traditional and novel measures. Epidemiology 2010;21:128-38.

36. Vickers AJ, Cronin AM. Everything you always wanted to know about evaluating prediction models (but were too afraid to ask). Urology 2010;76:1298-301.

37. D'Agostino RB, Nam B-H. Evaluation of the Performance of Survival Analysis models: discrimination and calibration measures. Handbook of Statistics 2003;23:1-25.

38. Grønnesby JK, Borgan O. A method for checking regression models in survival analysis based on the risk score. Lifetime Data Anal 1996;2:315-28

39. Hosmer DW, Lemeshow S, Sturdivant R, et al. Applied Logistic regression: Wiley, 2013.

40. Vickers AJ, Elkin EB. Decision curve analysis: a novel method for evaluating prediction models. Med Decis Making 2006;26:565-74. 